\title{
THE FLOW OF AN IDEAL INCOMPRESSIBLE FLUID ABOUT A LENS*
}

\author{
BY \\ MAX SHIFFMAN (New York University) AND D. C. SPENCER (Stanford University)
}

1. Introduction. The irrotational flow produced in an ideal incompressible fluid by the motion of an immersed object is described by a velocity potential function satisfying certain boundary conditions. For three-dimensional flows the velocity potential has been determined explicitly only for a very limited category of geometrically prescribed objects. In the present paper, we shall add to this list by constructing the flow about a lenticular object formed by two intersecting spheres, typical examples of which are illustrated in Figs. 1a-c. This lens flow is closely related to the well known case of two completely separated spheres, which might from a general point of view be considered as two spheres having an imaginary circle of intersection. In fact, it is this relationship which forms the point of departure of the present work and which will be clarified herein.

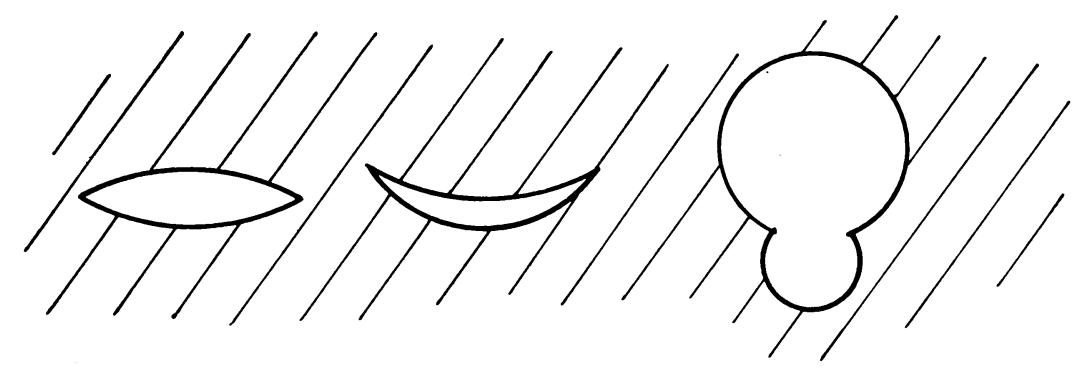

FIG. 1a

FIG. 1b

FIG. 1c

We shall consider, then, a lens formed by two intersecting spheres submerged in an ideal incompressible fluid and moving at unit velocity in the direction of its axis of symmetry. The velocity potential for the analogous two-dimensional problem could be easily determined by the use of conformal mapping but th is is of no avail for three dimensions. Rather, we shall continue the potential function $\Phi$ analytically as far as possible across the two faces of the lens, and determine its singularities. The extended function $\Phi$ is then defined over a multi-sheeted-in general infinite-sheeted-Riemann space with the sharp rim of the lens, the circle of intersection of the two spheres, as the branch line around which the Riemann space winds. By the nature of the analytic consideration, the singularities are located not only at the inverted points but also at the points at infinity in the various sheets. It is then necessary to construct fundamental potential functions defined on the multisheeted Riemann space and having prescribed singularities at given points. For this construction we shall use the same type of procedure as was introduced by A. Sommerfeld in his classical work on the theory of diffraction, where a similar question was considered for reflection across plane surfaces.

* Received Sept. 27, 1946. This paper is essentially an excerpt from a report by the authors entitled "The Force of Impact on a Sphere Striking a Water Surface," AMP Report 42.1R, Feb. 1945, Appl. Math. Group at New York University, prepared for the Office of Scientific Research and Development. The application to impact will be published separately. 
2. Procedure for separated spheres. Let $\Phi$ be the velocity potential (we use the convention that velocity $=-\operatorname{grad} \Phi)$. It satisfies the Laplace equation

$$
\Phi_{x x}+\Phi_{y y}+\Phi_{z z}=0,
$$

vanishes and is regular at $\infty$, and satisfies the following boundary condition: on the surface of the lens, the negative of the normal derivative of $\Phi$ is equal to the component of the velocity of the lens along its normal, or

$$
-\frac{\partial \Phi}{\partial n}=\cos \lambda \text {. }
$$

To see how to construct the potential function $\Phi$, let us first consider the case in which the two spheres forming the lens do not intersect and are thus completely separated (Fig. 2).

A classical procedure for finding the flow due to the vertical motion of the two spheres is by the method of images (see [1], p. 131 or [2], p. 420).* The flow produced by a single sphere alone moving in an infinite body of fluid is that created by a dipole of suitable strength placed at the center of the sphere. Thus, dipoles situated at $O$ and $O^{\prime}$ in the figure would individually give the correct flow if the influence of the other sphere could be neglected. But the dipole at $O^{\prime}$ destroys the correct boundary condition on the sphere $K$, and likewise for the dipole at $O$ and the sphere $K^{\prime}$. To remedy this, introduce the image of the dipole at $O^{\prime}$ relative to $K$ (and likewise for $O$ and $K^{\prime}$ ), namely, a dipole of suitable strength situated at the inverse point $Q_{1}$ (and at the inverse point $\left.Q_{1}^{\prime}\right)$. But again the dipole at $Q_{1}^{\prime}$ violates the boundary condition on $K$, etc. Indefinite continuation of this process leads to a convergent expression for the potential function which satisfies the boundary conditions on both spheres.

We shall apply a generalization of the above process to the lenticular case, when the spheres intersect. The process as it stands leads to a serious difficulty, namely that some of the suc-

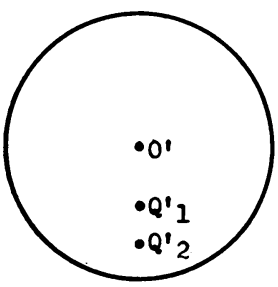
cessive images of the dipoles lie directly in the fluid, thus creating singularities inside the fluid which violate the requirements of the problem. For examples, in the particular case of Fig. 1a, the very first dipoles at the centers of the respective spheres already lie in the fluid. The difficulty may be overcome by using multivalued dipoles, the images of which lie in the various replicas ("sheets") of a Riemann space and do not insert singularities in that portion of the space in which we are interested. However, there is no difficulty in the special cases when the exterior angle between the intersecting spheres has the value $\pi / m$ where $m$ is an integer. This is discussed in App. A. It should of course be mentioned that when the exterior angle between the intersecting spheres is greater than $\pi$, as in Figs. 1a, b, the velocity of the fluid is infinite on the sharp rim of the lens.

3. Dipoles. We select the toroidal coordinate system $(\sigma, \psi, \theta)$ as most appropriate for our domain. These are formed from bipolar coordinates in the plane by rotation about an axis, and are defined by

* Numbers in brackets refer to the bibliography at the end of the paper. 


$$
x=r \cos \varphi, \quad y=r \sin \varphi, \quad r+i z=a i \cot \frac{1}{2}(\psi+i \sigma)
$$

where $x, y, z$ are rectangular coordinates, $r^{2}=x^{2}+y^{2}$, and $r, z$ can be expressed separately in terms of $\sigma, \psi$ by

$$
r=a \frac{\sinh \sigma}{s-\tau}, \quad z=a \frac{\sin \psi}{s-\tau}
$$

where

$$
s=\cosh \sigma, \quad \tau=\cos \psi .
$$

(See [2], p. 165.) Because of rotational symmetry, the angle $\theta$ will never enter. In the $r, z$-plane, the curve $\psi=$ constant is a circular arc connecting the points $+a$ and $-a$ on the $r$-axis, while $\sigma=$ constant is a circle with these two points as mutually inverse points. By letting $\psi$ vary from $-\infty$ to $+\infty$ without any identification, there results an infinite-fold Riemann space with the circle $r=a, z=0$ as branch line.

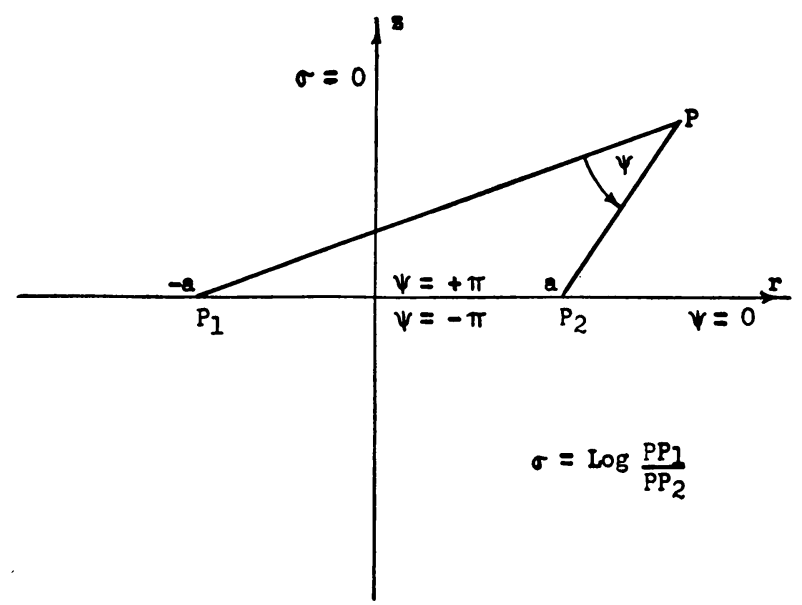

FIG. 3

The inverse distance between a point on the $z$-axis $\sigma=0$ at $\psi=\psi_{0}$ and an arbitrary point $(\sigma, \psi, \theta)$ is the absolute value of the quantity

$$
p\left(\psi_{0}\right)=\frac{\sin \left(\psi_{0} / 2\right)}{a} \sqrt{\frac{s-\tau}{s-\cos \left(\psi-\psi_{0}\right)}} .
$$

This represents a pole of strength \pm 1 at the point $\sigma=0, \psi=\psi_{0}$. We shall consider a dipole $q\left(\psi_{0}\right)$ of strength $a^{3}\left|\csc ^{3}\left(\psi_{0} / 2\right)\right| / 2$ situated at the same point, and pointing downwards, namely

$$
\begin{aligned}
q\left(\psi_{0}\right) & =-\frac{a^{3}}{2} \csc ^{3}\left(\psi_{0} / 2\right) \frac{\partial}{\partial z} p\left(\psi_{0}\right) \\
& =\frac{a}{2}\left\{\frac{(s-\tau)^{1 / 2} \sin \left(\psi-\psi_{0}\right)}{\left[s-\cos \left(\psi-\psi_{0}\right)\right]^{3 / 2}}+\frac{(s-\tau)^{1 / 2} \cot \left(\psi_{0} / 2\right)}{\left[s-\cos \left(\psi-\psi_{0}\right)\right]^{1 / 2}}\right\} .
\end{aligned}
$$

The Stokes' stream function $t\left(\psi_{0}\right)$ corresponding to $q\left(\psi_{0}\right)$ is (see [2], chapter XV, especially page 414$)$ : 


$$
t\left(\psi_{0}\right)=\frac{1}{2} a^{3} \csc ^{3}\left(\psi_{0} / 2\right) r^{2} p^{3}\left(\psi_{0}\right)=\frac{a^{2} \sinh ^{2} \sigma}{2(s-\tau)^{1 / 2}\left[s-\cos \left(\psi-\psi_{0}\right)\right]^{3 / 2}} .
$$

From this it follows that the potential function

$$
q\left(\psi_{0}\right)-q\left(\psi_{0}^{\prime}\right)
$$

has vanishing normal derivative on the spherical cap $\psi=c$ if

$$
\psi_{0}+\psi_{0}^{\prime}=2 c .
$$

For then $\dot{t}\left(\psi_{0}\right)-t\left(\psi_{0}^{\prime}\right)$ vanishes on $\psi=c$.

4. Multi-valued dipoles. The quantities $q(\xi), t(\xi)$ remain as potential and stream functions respectively of a flow if $\psi_{0}$ is replaced by a complex number $\xi$. Considering $\sigma, \psi$ temporarily as fixed, $q(\xi)$ and $t(\xi)$ as functions of $\xi$ in the complex $\xi$-plane are multi-valued and have branch points at the zeros of $s-\cos (\psi-\xi)$, that is at

$$
\xi=\psi \pm i \sigma+2 k \pi, \quad k=0, \pm 1, \pm 2, \cdots .
$$

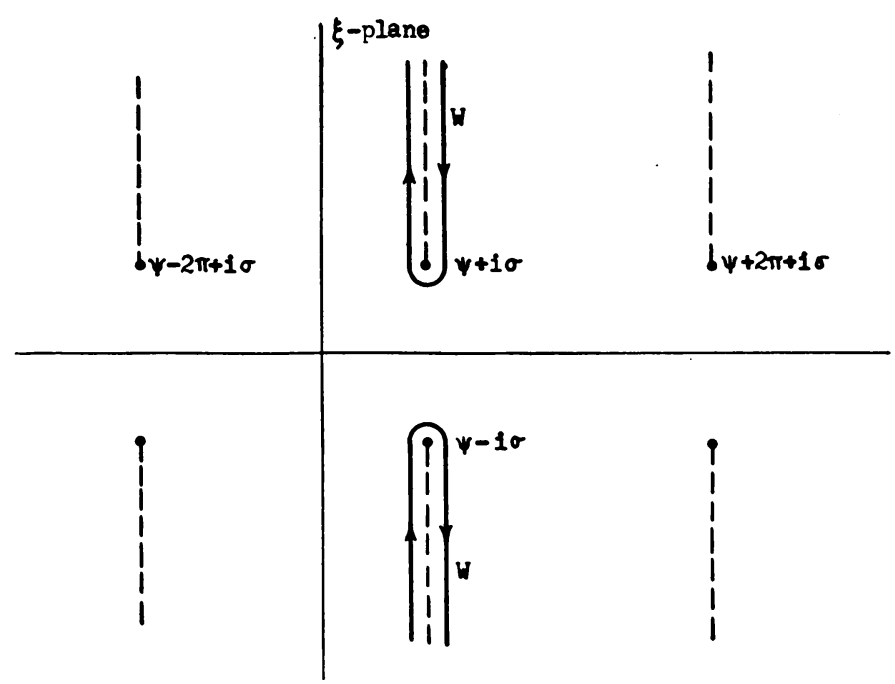

FIG. 4

For convenience, cut the $\xi$-plane by vertical lines proceeding from the branch points to $\pm i \infty$ as shown in Fig. 4 . The functions $q(\xi), t(\xi)$ are single-valued in this cut $\xi$-plane and take opposite signs on the opposite sides of each cut. We define $[s-\cos (\psi-\xi)]^{1 / 2}$ to be positive when $\xi$ is real.

The basic multi-value dipole $Q\left(\psi_{0}\right)$ situated at a (real) point $\sigma=0, \psi=\psi_{0}$ on the $z$-axis of our infinite sheeted Riemann space is defined as follows

$$
Q\left(\psi_{0}\right)=\frac{1}{2 \pi i} \int_{W} \frac{q(\xi)}{\xi-\psi_{0}} d \xi
$$

where the path $W$ of integration is the two-branched path indicated in Fig. 4 . The function $Q\left(\psi_{0}\right)$ is a potential function in $(\sigma, \psi)$ since differentiations can be performed under the integral sign, the path $W$ being temporarily fixed.

For an $n$-sheeted Riemann space in which values of the angle $\psi$ differing by $2 n \pi$ 
would be identified, the corresponding $n$-valued dipole $Q\left(\psi_{0}\right)$ is

$$
Q^{(n)}\left(\psi_{0}\right)=\frac{1}{2 \pi i} \int_{W} \frac{q(\xi)}{2 n} \cot \frac{\xi-\psi_{0}}{2 n} d \xi
$$

Since $\cot \left(\xi-\psi_{0}\right) / 2 n$ is periodic in $\xi$ of period $2 n \pi$, and $q(\xi)$ is periodic of period $2 \pi$, it is easy to see that $Q^{(n)}\left(\psi_{0}\right)$ is periodic in $\psi$ of period $2 n \pi$.

We now briefly describe the properties of $Q\left(\psi_{0}\right)$, a similar description applying to $Q\left(\psi_{0}\right)$ (see [3], [4] for a complete analysis, the following being but a bare sketch). On account of the pole of the integrand in (4.1) at $\psi_{0}$ one sees that

$$
Q\left(\psi_{0}\right)-q\left(\psi_{0}\right)
$$

is regular as $\sigma \rightarrow 0, \psi \rightarrow \psi_{0}$ (where $\psi_{0} \neq 2 j \pi$ ). Thus $Q\left(\psi_{0}\right)$ behaves like a dipole near $\sigma=0, \psi=\psi_{0}$, but is regular near $\sigma=0, \psi=\psi_{0}+2 k \pi, k= \pm 1, \pm 2, \cdots$ since the integrand is regular at these points.

In addition, there are still the poles of $q(\xi)$ itself at $\xi=2 k \pi, k=0, \pm 1, \pm 2, \ldots$ due to the presence of the term $\cot \xi / 2$ (see (3.1)). One easily sees that

$$
Q\left(\psi_{0}\right) \rightarrow \frac{a}{2 k \pi-\psi_{0}}
$$

as $\sigma \rightarrow 0$ and $\psi \rightarrow 2 k \pi, k=0, \pm 1, \pm 2, \cdots\left(\psi_{0} \neq 2 j \pi\right)$. Thus the function $Q\left(\psi_{0}\right)$ is not simply a multi-valued dipole at $\psi_{0}$ but it has certain non-vanishing values at $\infty$ $(\sigma=0, \psi=2 k \pi)$ in the various sheets and has no other singularities.

(A constant value at $\infty$ may be considered as a pole at $\infty$. If in the definition of $q(\xi)$ the term $\cot \xi / 2$ were replaced by $\cot \psi_{0} / 2$, the resulting function in (5.1) would be zero at the points at $\infty$; this would be a simple dipole. However, a simple dipole would lead to great difficulties, and our definition of $q(\xi)$ is in this case the correct generalization to the multi-sheeted case. This illustrates how careful one must be in the choice and analysis of the singularities, with special reference to the behavior at infinity.)

For the special case in which $\psi_{0}$ is a multiple of $2 \pi$, the integrand in (4.1) has a double pole. For example, $Q(0)$ has an integrand in (4.1) which has a double pole at $\xi=0$, and the residue there is

$$
\frac{a}{2} \frac{\sin \psi}{s-\tau}+a\left[\frac{\partial}{\partial \xi}\left(\frac{s-\tau}{s-\cos (\psi-\xi)}\right)^{1 / 2}\right]_{\xi=0}=a \frac{\sin \psi}{s-\tau}=z .
$$

Therefore

$$
Q(0)-z \rightarrow 0 \text { as } \sigma \rightarrow 0, \psi \rightarrow 0
$$

and so $Q(0)$ behaves like $z$ near the point at $\infty$ in the first sheet. A similar analysis applies to $Q(2 k \pi)$.

In the $n$-sheeted case, it is of interest to consider the special case $n=1$ where we are in the original single-sheeted space. The integrand in (4.2) is then of period $2 \pi$, and we have

$$
Q^{(1)}\left(\psi_{0}\right)=q\left(\psi_{0}\right)-a / 2 \cot \left(\psi_{0} / 2\right), \quad \psi_{0} \neq 0,
$$

so $Q^{(1)}\left(\psi_{0}\right)$ differs from an ordinary dipole $q\left(\psi_{0}\right)$ by a constant. If in (4.4) we now let 
$\psi_{0} \rightarrow 0$, we obtain

$$
Q^{(1)}\left(\psi_{0}\right) \rightarrow z \text { as } \psi_{0} \rightarrow 0 .
$$

It may also be verified directly that

$$
Q^{(1)}(0)=z .
$$

5. Inversion of multi-valued dipoles. It is well known that in ordinary space the image of a dipole with respect to a sphere is another dipole situated at the inverse point. In this section the theorem will be extended to multi-sheeted spaces.

THEOREM 5.1. The function $Q\left(\psi_{0}\right)-Q\left(\psi_{0}^{\prime}\right)$ has vanishing normal derivative on the spherical cap $\psi=$ constant $=c$ if $\psi_{0}+\psi_{0}^{\prime}=2 c$.

Proof. The stream function $T\left(\psi_{0}\right)$ corresponding to $Q\left(\psi_{0}\right)$ is

$$
T\left(\psi_{0}\right)=\frac{1}{2 \pi i} \int_{W} \frac{t(\xi)}{\xi-\psi_{0}} d \xi
$$

where $t(\xi)$ is the stream function corresponding to $q(\xi)$, obtained from (3.2) by replacing $\psi_{0}$ by $\xi$. It is required to show merely that $T\left(\psi_{0}\right)-T\left(\psi_{0}^{\prime}\right)$ is constant on $\psi=c$.

Make the substitution $\xi-\psi=\zeta$. Then

$$
\begin{aligned}
t(\xi) & =\frac{a^{2}}{2} \frac{\sinh ^{2} \sigma}{(s-\tau)^{1 / 2}(s-\cos \zeta)^{3 / 2}} \\
T\left(\psi_{0}\right) & =\frac{1}{2 \pi i} \int_{W} \frac{t(\xi)}{\zeta+\psi-\psi_{0}} d \zeta
\end{aligned}
$$

where $W^{\prime}$ is the path in the $\zeta$-plane shown in Fig. 5. By the symmetry of the path $W^{\prime}$, the lower path $W_{l}^{\prime}$ is obtained from the upper path $W_{u}^{\prime}$ by replacing $\zeta$ by $-\zeta$. The

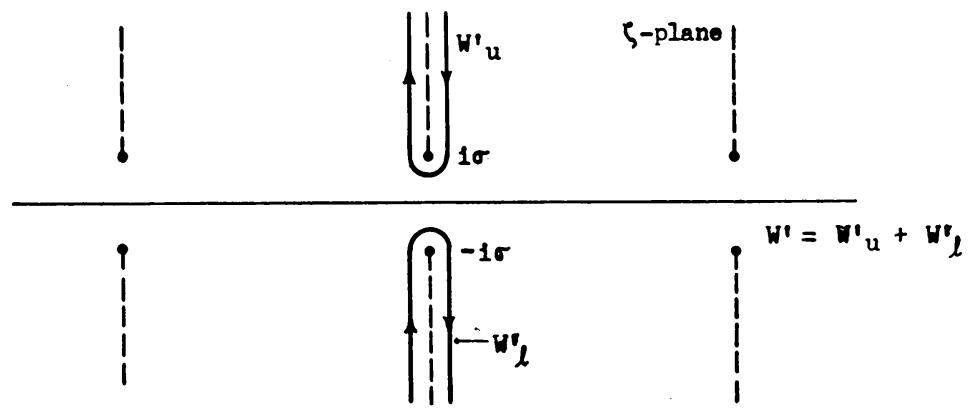

FIG. 5

function $t(\xi)$ is by (5.2) an even function of $\zeta$, and (5.3) can be written in the form

$$
T\left(\psi_{0}\right)=\frac{1}{2 \pi i} \int_{W^{\prime} u} t(\xi)\left(\frac{1}{\zeta+\left(\psi-\psi_{0}\right)}+\frac{1}{\zeta-\left(\psi-\psi_{0}\right)}\right) d \zeta
$$

Likewise for $T\left(\psi_{0}^{\prime}\right)$. Evaluating at $\psi=c$, and noting that the hypothesis $\psi_{0}+\psi_{0}^{\prime}=2 c$ can be written $c-\psi_{0}=-\left(c-\psi_{0}^{\prime}\right)$, eq. (5.4) shows immediately that $T\left(\psi_{0}\right)-T\left(\psi_{0}^{\prime}\right)=0$ when $\psi=c$, and the theorem is proved. 
THEOREM 5.2. The function $Q\left(\psi_{0}\right)+Q\left(-\psi_{0}\right)$ vanishes on $\psi=0$. In particular $Q(0)$ vanishes on $\psi=0$.

Proof. For $\psi=0$ the function $q(\xi)$ is an odd function of $\xi$ by (3.1). The same argument as above yields $Q\left(\psi_{0}\right)=-Q\left(-\psi_{0}\right)$ for $\psi=0$.

6. Construction of the potential function for a symmetrical lens. We are now prepared to apply the method of images described in Sec. 2. For the sake of convenience we shall first consider the case of a symmetrical lens, the two spherical portions having the equation $\psi=\beta$ and $\psi=-\beta$ where the exterior angle between the two intersecting spheres is $2 \beta$. The radius of the circle of intersection of the spheres is $a$, the semi-thickness of the lens is $B$, and the radius of the two symmetrical spheres is $A$, as indicated in Fig. 6. The motion of the lens is in the negative $z$-direction.

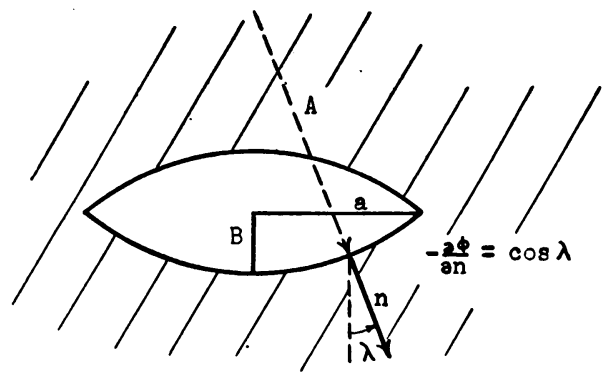

FIG. 6

Let $\Phi$ be the desired potential function, and set

$$
V=\Phi-z .
$$

Then $V$ is a potential satisfying the following conditions

$$
\begin{aligned}
& -\frac{\partial V}{\partial n}=0 \text { on } \psi=\beta \\
& -\frac{\partial V}{\partial n}=0 \text { on } \psi=-\beta
\end{aligned}
$$

and

$$
V \text { behaves like }-z \text { at } \infty \text {. }
$$

The potential function $V$ corresponds to the flow about a fixed lens, the fluid having unit velocity at $\infty$.

We shall construct $V$ by the method of images, using the infinite sheeted Riemann space described by $0 \leqq \sigma \leqq \infty,-\infty<\psi<\infty$. (It may be pointed out that if the angle $\beta=n \pi / 2 q$ where $n, q$ are integers, it would be sufficient to use an $n$-sheeted Riemann space in place of the infinite sheeted one.) First start with $-Q(0)$ which has the correct behavior (6.4) as $\sigma \rightarrow 0, \psi \rightarrow 0$ (by (4.3)). To satisfy (6.2), one must add $Q(2 \beta)$ by theorem (5.1); and to satisfy (6.3) one must add $Q(-2 \beta)$. But $Q(-2 \beta)$ destroys the correct boundary condition on $\psi=\beta$, and likewise for $Q(2 \beta)$ on the spherical cap $\psi=-\beta$. To remedy this one must add $-Q(4 \beta)$ by Theorem 5.1 , and likewise $-Q(-4 \beta)$. Continuing this process indefinitely, one obtains

$$
V=\sum_{k=-\infty}^{\infty}(-1)^{k+1} Q(2 k \beta) .
$$

The convergence of this sum follows directly from its evaluation below.

We may write (6.5) in the forms

$$
V=\sum_{k=0}^{\infty}(-1)^{k+1}[Q(-2 k \beta)-Q(2(k+1) \beta)]
$$




$$
V=\sum_{k=0}^{\infty}(-1)^{k+1}[Q(2 k \beta)-Q(-2(k+1) \beta)] .
$$

By virtue of Theorem 5.1, eqs. (6.6) and (6.7) show that (6.2) and (6.3) are satisfied respectively. From the appearance of $-Q(0)$ in (6.5) we see that (6.4) is also satisfied.

Substituting from (4.1) we obtain

$$
V=\frac{1}{2 \pi i} \int_{W} q(\xi) \sum_{k=-\infty}^{\infty}(-1)^{k+1} \frac{1}{\xi-2 k \beta} d \xi
$$

or

$$
V=-\frac{1}{2 \pi i} \int_{W} q(\xi) \frac{\pi}{2 \beta} \csc \frac{\pi \xi}{2 \beta} d \xi
$$

For the desired potential function $\Phi$, we have from (6.1) and (4.5),

$$
\Phi=-\frac{1}{2 \pi i} \int_{W} q(\xi)\left(\frac{\pi}{2 \beta} \csc \frac{\pi \xi}{2 \beta}-\frac{1}{2} \cot \frac{\xi}{2}\right) d \xi .
$$

Formulas (6.8) or (6.9) are the final expressions for $V$ or $\Phi$ respectively.

The integrals may be expressed in real form by integrating by parts and then moving the path $W$ into the cuts which it surrounds. The results are

$$
V=\frac{A \sin \beta}{2 \beta} \int_{\sigma}^{\infty} \sqrt{\frac{s-\tau}{\cosh u-s}} \operatorname{Im}\left[\cot \frac{\xi}{2} \csc \frac{\pi \xi}{2 \beta}+\frac{\pi}{\beta} \csc \frac{\pi \xi}{2 \beta} \cot \frac{\pi \xi}{2 \beta}\right] d u
$$

where $\xi=\psi+i u$, and an analogous expression for $\Phi$.

7. Virtual mass of the fluid. The kinetic energy of the fluid is $(M / 2) \times$ (velocity of object) ${ }^{2}$, where

$$
M=\rho \iiint(-\operatorname{grad} \Phi)^{2} d x d y d z=-\iint_{\text {lens }} \Phi \frac{\partial \Phi}{\partial n} d S,
$$

and $\rho$ is the density of the fluid, $n$ is a normal directed into the fluid. The contribution due to a surface integral over a surface which approaches $\infty$ vanishes. Using the boundary condition for $-\partial \Phi / \partial n$ on the surface of the lens,

$$
M=\rho \iint_{\text {lens }} \Phi \cos \lambda d S,
$$

where $\lambda$ is the angle between the normal and the direction of motion, indicated in Fig. 6. This quantity $M$ is called the "virtual mass" or the "added mass" of the fluid. The same formula (7.1) is obtained if the virtual mass is based on the vertically downward momentum of the fluid.

The virtual mass $M$ is more easily calculated by obtaining a different form for (7.1).* Formula (7.1) can be written (motion is in the negative $z$-direction)

$$
M=-\rho \iint_{\text {lens }} \Phi \frac{\partial z}{\partial n} d S,
$$

* The longer calculation required in (7.1) was carried out in the original OSRD report with the same result. 
since $-\partial z / \partial n=\cos \lambda$. Green's formula,

$$
\iint\left(u \frac{\partial v}{\partial n}-v \frac{\partial u}{\partial n}\right) d S=0,
$$

can be applied to the two potential functions $\Phi, z$ yielding

$$
M=-\rho \iint_{\text {lens }} z \frac{\partial \Phi}{\partial n} d S+\rho \iint_{\infty}\left(\Phi \frac{\partial z}{\partial n}-z \frac{\partial \Phi}{\partial n}\right) d S
$$

where the last integral is taken over a surface which approaches $\infty$, for example a sphere of radius $R$ where $R \rightarrow \infty$. At $\infty, \Phi$ behaves like a dipole and we may set

$$
\Phi \sim D \cdot \cos \theta / R^{2} \text { at } \infty
$$

where $(\cos \theta) / R^{2}$ is a unit dipole at th origin pointing in the direction of motion of the lens, and $D$ is the strength of the dipole equivalent ta $\infty$ to $\Phi$. Then on a sphere of radius $R, z=-R \cos \theta$, and a simple calculation gives $4 \pi \rho D$ as the value of the second integral in (7.2).

For the first integral in (7.2) over the surface of the object, we have

$$
-\frac{\partial \Phi}{\partial n}=\cos \lambda
$$

and

$$
-\rho \iint_{\text {lens }} z \frac{\partial \Phi}{\partial n} d S=\rho \iint z \cos \lambda d S=-\rho \cdot \text { volume of lens }
$$

since $\cos \lambda d S= \pm d x d y$, the sign depending on the sign of $\cos \lambda$. Substitution of both evaluations into (7.2) yields the final formula, valid for any symmetrical object moving in an infinite fluid :*

$$
M=4 \pi \rho D-\text { mass of fluid displaced by object }
$$

where $D$ is the "dipole strength" at $\infty$ of the potential function $\Phi$ as defined in (7.3).

It remains to evaluate the dipole strength $D$ for the particular lens flow. The dipole $q(\xi)$ from its very construction has strength

$$
a^{3}\left|\csc ^{3}(\xi / 2)\right| / 2
$$

where $\left|\csc ^{3}(\xi / 2)\right|$ is the piecewise analytic function of the complex variable $\xi$ equal to $\pm \csc ^{3}(\xi / 2)$, the sign being determined in such a way that $\left|\csc ^{3}(\xi / 2)\right|$ is positive for real $\xi$ (there are vertical cuts extending from $2 n \pi-i \infty$ to $2 n \pi+i \infty$ ). This may also be verified directly from (3.1) or (3.2).

The dipole strength of $\Phi$ can now be obtained from (6.9). The integrand has no singularity near $\xi=0$ or near $\xi=\psi$ (we are considering $\psi \rightarrow 0$ ), so that the path $W$ may be deformed into the path $W^{\prime \prime}$ indicated in Fig. 7a. Inserting the dipole strength (7.5) of $q(\xi)$, one obtains for the dipole strength $D$ of $\Phi$ at $\infty$ :

$$
D=-\frac{1}{2 \pi i} \int_{W^{\prime \prime}} \frac{a^{3}}{2}\left|\csc ^{3}(\xi / 2)\right|\left(\frac{\pi}{2 \beta} \csc \frac{\pi \xi}{2 \beta}-\frac{1}{2} \cot \frac{\xi}{2}\right) d \xi
$$

* The same formula applies for any (asymmetrical) object but then $D$ is the component of the dipole strength at $\infty$ in the direction of motion. 
where now the diagram in the $\xi$-plane is indicated by Fig. 7b. The second term in the integrand involving cot $\xi / 2$ can be integrated explicitly and has the value zero. Further, the path $W^{\prime \prime}$ reduces to $W_{\imath}^{\prime \prime}$ by replacing $\xi$ by $-\xi$. Making the change of variable $\pi \xi / 2 \beta=z$, we have

$$
D=\frac{a^{3}}{2 \pi i} \int_{L} \csc ^{3} \frac{\beta z}{\pi} \csc z d z
$$

where the path $W_{l}^{\prime \prime}$ is henceforth denoted by $L$ and is a vertical line slightly to the left of the imaginary axis.

The virtual mass can now be obtained from (7.4), (7.7) and

$$
\text { mass of displaced fluid }=\frac{8}{3} \pi \rho A^{3}(2-\cos \beta) \cos ^{4} \beta / 2 \text {. }
$$

The integral in (7.7) is evaluated for angles $\beta$ which are rational multiples of $\pi$ in Sec. 9. The results for the virtual mass as a function of the thickness of the lens are exhibited in dimensionless form in Figs. 8 and 9. In Fig. 8 we have plotted $M / \pi \rho A^{3}$ as a function of $B / A$, where $A$ is the radius of the spheres bounding the lens and $B$ is the half thickness of the lens. The case of completely separated spheres, $B / A>2$, is included in the graph (see App. C). It is also

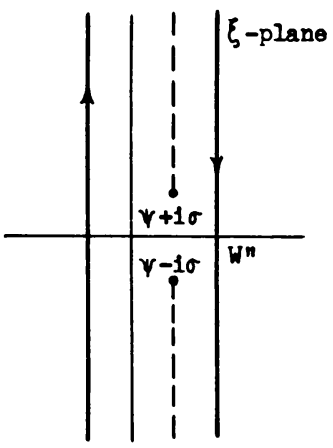

FIG. 7a

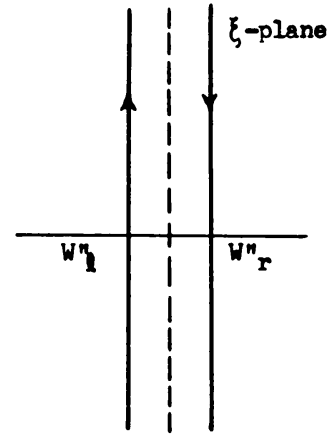

FIg. 7b of interest to emphasize the presentation surface of the lens, and for this purpose we have plotted $M / \pi \rho a^{3}$ as a function of $B / a$ in Fig. 9, where $a$ is the radius of the sharp circular rim of the lens. For $B / a>1$, the lens has a figure eight cross section and the presentation radius is preferably $A$ instead of $a$, so that Fig. 8 should be used for this range.

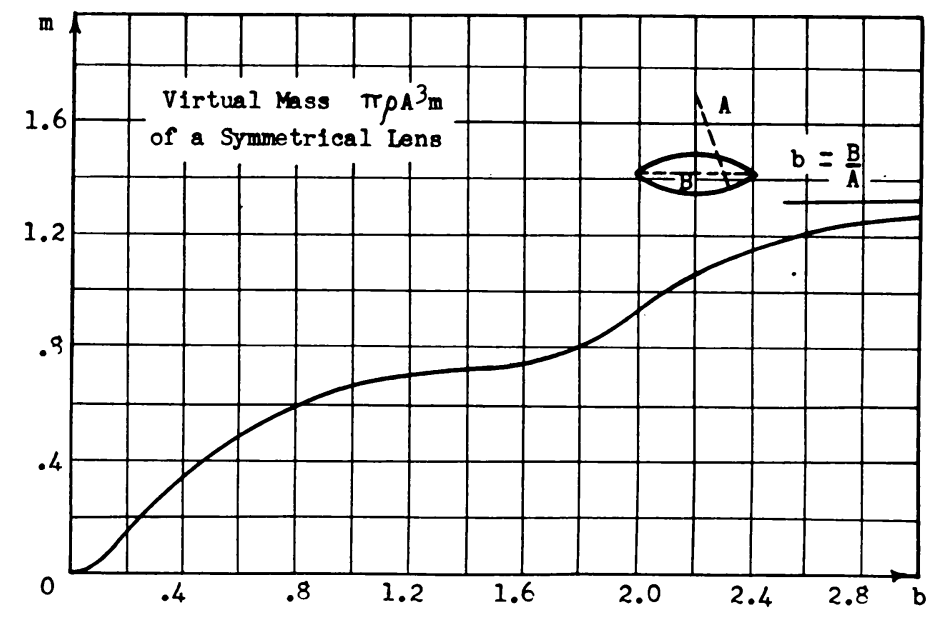

FIG. 8 


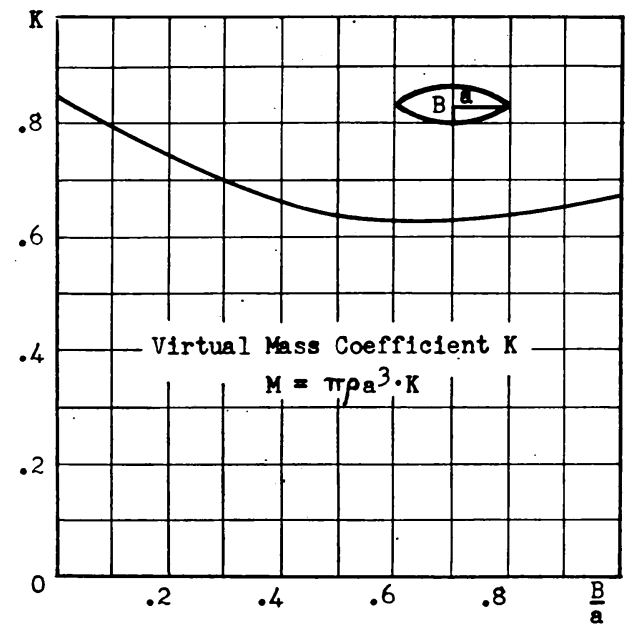

FIG. 9

\section{Potential function and virtual mass} for an arbitrary lens. We construct the velocity potential of the flow around an asymmetrical lens bounded by the spherical caps $\psi=\alpha-\beta$ and $\psi=-\beta$, where $\beta$ and $\alpha-\beta$ are both positive. We suppose that the lens is immersed in an infinite fluid and that it is moving downward (i.e. in the negative $z$-direction) with unit velocity. The symmetrical lens is obtained by taking $\alpha=2 \beta$, and this is the case which has been discussed in the main body of the paper.

The potential function describing the flow satisfies conditions (6.1)-(6.4) as in Sec 6 , but. in place of (6.2), we have

$$
-\frac{\partial V}{\partial n}=0 \quad \text { on } \quad \psi=\alpha-\beta \text { and on } \psi=-\beta .
$$

In order to illustrate a remark made in Sec. 6 , namely that the $n$-fold Riemann space may be used if the intersection angle, here equal to $\alpha$, is rational, we suppose that $\alpha=n \pi / m$. The formulas which we obtain are then valid for all $\alpha$ by continuity.

The solution is the potential function

$$
\begin{aligned}
V & =\sum_{k=1}^{m}\left[Q^{(n)}(2 k \alpha-2 \beta)-Q^{(n)}(-2 k \alpha)\right] \\
& =\sum_{k=1}^{m}\left[Q^{(n)}(2(k+1) \alpha-2 \beta)-Q^{(n)}(-2 k \alpha)\right]
\end{aligned}
$$

making use of the fact that $Q^{(n)}\left(\psi_{0}\right)$ is periodic in $\psi_{0}$ of period $2 n \pi$. The first form of (8.2) shows by Theorem 5.1 that $V$ has vanishing normal derivative on $\psi=-\beta$, while the second form yields the vanishing of the normal derivative on $\psi=\alpha-\beta$. Furthermore, at $\infty$ in the first sheet $(\sigma=0, \psi=0)$, all the terms in the summation are regular except $-Q^{(n)}(-2 m \alpha)=$ $-Q^{(n)}(-2 n \pi)=-Q^{(n)}(0)$ which behaves at $\infty$ like $-z+0(1)$ (compare (4.3)). Substituting from (4.2) into (8.2) and evaluating the sums, we obtain the formula

$$
V=\frac{1}{2 \pi i} \int_{W} q(\xi) \frac{\pi}{2 \alpha}\left(\cot \frac{\pi(\xi+2 \beta)}{2 \alpha}-\cot \frac{\pi}{2 \alpha}\right) d \xi .
$$

The desired potential function $\Phi=V+z$.

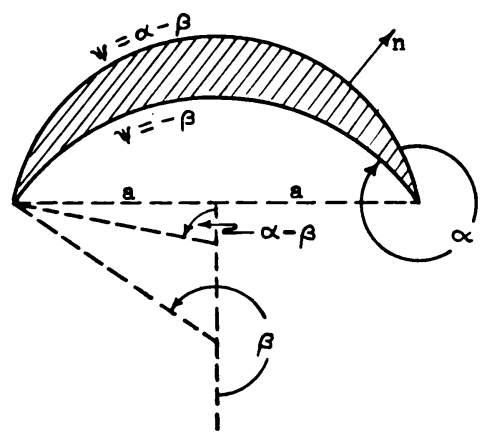

FIG. 10

Formula (8.3) reduces to the old formula (6.8) in the special case when $\alpha=2 \beta$. It is interesting to note that (8.3) is built out of two terms of the type 


$$
\bar{Q}\left(\psi_{0}\right)=\frac{1}{2 \pi i} \int_{W} q(\xi) \frac{\pi}{2 \alpha} \cot \frac{\pi\left(\xi-\psi_{0}\right)}{2 \alpha} d \xi .
$$

According to Theorem 5.1, the image of $-\bar{Q}(0)$ relative to $\psi=-\beta$ is $\bar{Q}(-2 \beta)$, while its image relative to $\psi=\alpha-\beta$ is $\bar{Q}(2 \alpha-2 \beta)$. But $\bar{Q}(-2 \beta)=\bar{Q}(2 \alpha-2 \beta)$ since $\bar{Q}\left(\psi_{0}\right)$ is periodic in $\psi_{0}$ of period $2 \alpha$. Thus the potential function

$$
V=-\bar{Q}(0)+\bar{Q}(-2 \beta)
$$

satisfies the boundary conditions on both $\psi=-\beta$ and $\psi=\alpha-\beta$, and has the required behavior at $\infty$. It is therefore the solution to our problem. This is exactly (8.3).

The expression $\bar{Q}\left(\psi_{0}\right)$ in (8.4) might be interpreted as a fundamental dipole in a space obtained by identifying angles $\psi$ wh ich differ by $2 \alpha$. This would be analogous to the two-dimensional case in which $V$ could be extended across $\psi=-\beta$ into the region between $\psi=-\beta$ and $\psi=-\alpha-\beta$ by inversion and then noting that this extension is likewise the extension across $\psi=\alpha-\beta$ if one identifies $\psi=\alpha-\beta$ with $\psi=-\alpha-\beta$. But the simple extension by inversion does not apply in the three-dimensional case but rather a more complicated continuation. In fact $\bar{Q}\left(\psi_{0}\right)$ in (8.4) is not single valued in the space obtained by identifying angles $\psi$ which differ by $2 \alpha$. Thus the analogy with the two-dimensional case given by formulas $(8.4),(8.5)$ is not exact, but is nevertheless noteworthy.

The calculation of the virtual mass is similar to that of Sec. 7. The result is

$$
M=M_{1}-M_{2}
$$

where

$$
M_{1}=2 \pi \rho a^{3} \sin \frac{\pi \beta}{\alpha} \frac{1}{2 \pi i} \int_{L} \csc ^{3} \frac{\alpha z}{\pi} \csc z\left\{\csc \left(z+\frac{\pi \beta}{\alpha}\right)-\csc \left(z-\frac{\pi \beta}{\alpha}\right)\right\} d z
$$

and where

$$
\begin{aligned}
M_{2}=\frac{1}{6} \pi \rho a^{3}[(2-\cos \beta) \cot & \frac{\beta}{2} \csc ^{2} \frac{\beta}{2} \\
& \left.+(2-\cos (\alpha-\beta)) \cot \frac{\alpha-\beta}{2} \csc ^{2} \frac{\alpha-\beta}{2}\right]
\end{aligned}
$$

is the mass of the fluid displaced by the lens.

9. The symmetrical lens. Virtual mass in special cases. (i) For the determination of the virtual mass there remains but to evaluate the integral in (7.7), and this can be done in terms of residues when the angle $\beta$ is a rational multiple of $\pi$. Suppose that

$$
\beta=\frac{n \pi}{q}
$$

where $n$ and $q$ are relatively prime integers and $n<q$. The integral to be evaluated is then

$$
I=\frac{1}{2 \pi i} \int_{L} \csc ^{3} \frac{n z}{q} \csc z d z
$$

where we repeat that $L$ is a vertical line slightly to the left of the imaginary axis. 
Let $\delta$ be a fixed positive number and introduce the factor $e^{-\delta z}$ in the integrand in (9.2). Then

$$
\begin{aligned}
\frac{1}{2 \pi i} \int_{L} \csc ^{3} \frac{n z}{q} \csc z e^{-\delta z} d z & =- \text { sum of residues to the right of } L \\
& =-\frac{1}{1-(-1)^{n+q} e^{-\delta q \pi}} \times \begin{array}{l}
\text { sum of residues } \\
\text { between } 0 \text { and } q \pi .
\end{array}
\end{aligned}
$$

Calculating the residues between 0 and $q \pi$, letting $\delta \rightarrow 0$ and observing that the coefficient of $1 / \delta$ must vanish, we obtain two different results according as $n+q$ is odd or even. If $n+q$ is odd,

$$
\begin{aligned}
I=\frac{1}{2}\left[\sum_{j=1}^{q-1}(-1)^{j-1} \csc ^{3} \frac{j n \pi}{q}\right. & \\
& \left.+\frac{q}{2 n} \sum_{k=1}^{n-1}(-1)^{k-1}\left\{\csc z+\frac{q^{2}}{n^{2}} \frac{d^{2}}{d z^{2}}(\csc z)\right\}_{z=k q \pi / n}\right],
\end{aligned}
$$

and if $n+q$ is even,

$$
\begin{aligned}
I= & \frac{1}{q \pi}\left[\left(\frac{q^{3}}{6 n^{3}}+\frac{q}{2 n}\right)+\pi \sum_{j=1}^{q-1}(-1)^{j} j \csc ^{3} \frac{j n \pi}{q}\right. \\
& \left.+\frac{q}{2 n} \sum_{k=1}^{n-1}(-1)^{k}\left\{z \csc z+\frac{q^{2}}{n^{2}} \frac{d^{2}}{d z^{2}}(z \csc z)\right\}_{z=k q \pi / n}\right] .
\end{aligned}
$$

The computations based on these formulas were used in plotting the graph of Fig. 8 .

It is interesting to note that formulas (9.4), (9.5) involve angles which are multiples of $q \pi / n$ as well as those which are multiples of $n \pi / q$. These trigonometric sums, which would normally depend in a highly discontinuous manner on the angle $\beta=n \pi / q$, do indeed depend continuously since they are special cases of the integral (9.2).

For the purpose of checking, it is worth while to calculate the virtual mass in certain easy special cases where it can be calculated by other means.

(ii) If $\beta=\pi / 2$, eq. (9.4) for $n=1, q=2$ gives $I=1 / 2$, or from (7.4), (7.7), (7.8)

$$
M=\frac{2}{3} \pi \rho A^{3} \text {. }
$$

This trivial case corresponds to the flow about a sphere in an infinite fluid, and the virtual mass is then known to be as stated.

(iii) If we let $\beta \rightarrow 0$, we obtain from (7.7) and the fact that $a=A \sin \beta$,

$$
D \rightarrow \frac{\pi^{3} A^{3}}{2 \pi i} \int_{L} \frac{\csc z}{z^{3}} d z=A^{3} \sum_{j=1}^{\infty}(-1)^{j-1} / j^{3} .
$$

Substituting in (7.4), (7.7), (7.8), the virtual mass of the fluid, when the two spheres are tangent $(\beta=0)$, is

$$
M=4 \pi \rho A^{3}\left[\sum_{j=1}^{\infty}(-1)^{j-1} / j^{3}-2 / 3\right] .
$$

This value agrees with the limit obtained when completely separated spheres ap- 
proach tangency (see App. C) and so is a consequence of known formulas (see [2], p. 447).

(iv) Taking $\beta=\pi$, the virtual mass $M$ is obviously zero. However if we keep $a$ (instead of $A$ ) as fixed, then we obtain the virtual mass for a circular disc of radius $a$. Taking $\beta=\pi$ in (7.4), (7.7), (7.8), we obtain

$$
M=\frac{4 \pi \rho a^{3}}{2 \pi i} \int_{L} \csc ^{4} z d z=\frac{8}{3} \rho a^{3} .
$$

This is also a known result (see [2], p. 428).

10. The symmetrical lens. Derivative of the virtual mass. For the application of the lens flow to the impact of a sphere on a fluid surface, it is necessary to calculate

$$
\frac{d m}{d b} \text { where } m=M / \pi \rho A^{3}, \quad b=B / A .
$$

This can be done by differentiating formulas (7.4), (7.7), (7.8), leading to another trigonometric contour integral. This integral can then be evaluated for $\beta=n \pi / q$ as in the preceding section, with two cases appearing, according as $n+q$ is odd or even. We shall not write down these formulas since they are complicated and are similar to those in the preceding section. Just the remark should be made that in equating the coefficient of $1 / \delta$ to zero one obtains evaluations of certain trigonometric sums, involving angles which are multiples of $q \pi / n$ and $n \pi / q$, which appear at first glance quite startling.

On the basis of these formulas, a graph of $d m / d b$ was prepared and is shown in Fig. 11.

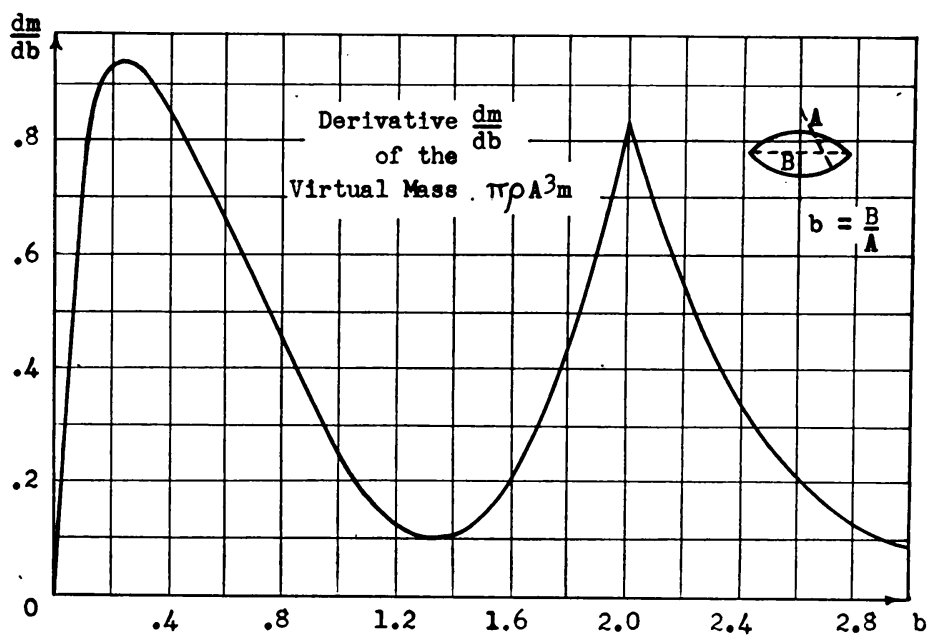

Fig. 11

\section{Appendix A}

Figure eight lens with exterior angle $\alpha=\pi / m$. The elementary process of successive images of dipoles fails in general without the introduction of multi-sheeted spaces. But this method succeeds for the special case of a lens with exterior angle $\alpha=\pi / m$, 
in which case the procedure described in Sec. 2 terminates after $m$ steps. On the $m$ th step, the same dipole corrects the boundary conditions on both spheres $K$ and $K^{\prime}$ simultaneously, and the total number of dipoles is $2 m-1$. An example is a figure eight lens with $\alpha=\pi / 2$, which has three dipoles situated at the points $O, O^{\prime}, Q$ indicated in Fig. 12. (By inversion relative to $K$ or to $K^{\prime}$, the circle $O R O^{\prime} S$ inverts into the straight line $R Q S$. Thus $Q$ is the inverse of $O^{\prime}$ relative to $K$ and also of $O$ relative to $K^{\prime}$.) The dipoles are directed vertically downward and their strengths at $O, O^{\prime}$ and $Q$ are $A^{3} / 2, A^{\prime 3} / 2$ and $-A^{3} A^{\prime 3} / 2\left(\overline{O O}^{\prime}\right)^{3}=-a^{3} / 2$ respectively.

For the general case in which $\alpha=\pi / m$, it is convenient to use the angular coordinate $\psi$. Let the equations of the two spherical caps $K$ and $K^{\prime}$ be $\psi=-\alpha / 2+\gamma$ and $\psi=\alpha / 2+\gamma$ respectively, where $-\alpha / 2<\gamma<\alpha / 2$. The dipoles are situated on the axis of symmetry at the points

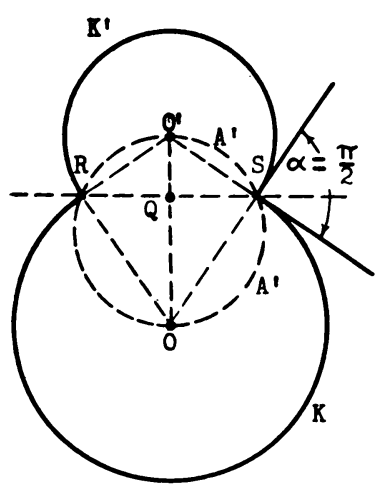

FIG. 12

$$
\begin{aligned}
& P_{\nu} ; \quad \psi_{\nu}=-(\nu+1) \alpha+\left[1+(-1)^{\nu}\right] \gamma, \quad \nu=0, \cdots, m-1 \\
& P_{\nu}^{\prime} ; \quad \psi_{\nu}^{\prime}=(\nu+1) \alpha+\left[1+(-1)^{\nu}\right] \gamma, \quad \nu=0, \cdots, m-1 .
\end{aligned}
$$

The points $P_{m-1}$ and $P_{m-1}^{\prime}$ are identical, since $\psi_{m-1}^{\prime}-\psi_{m-1}=2 \pi$. The strengths of the dipoles at $P_{\nu}$ and $P_{\nu}^{\prime}$ are $(-1)^{\nu-1} \bar{S} \bar{P}_{\nu}^{3} / 2$, where $S$ is a point on the sharp circular rim of intersection of the two spherical caps and $\bar{S}_{\nu_{\nu}}$ is the distance from $P_{\nu}$ to $S$.

The expression for the velocity potential is now easily written down in terms of these $(2 m-1)$ dipoles, and the virtual mass obtained from formula (7.4).

\section{Appendix B}

Spherical shell and figure eight lens with exterior angle $\alpha=2 \pi / m$. In the special case when the exterior angle $\alpha=2 \pi / m$, the velocity potential $V$ (or $\Phi$ ) can be expressed in terms of elementary functions. According to Sec. 8 all that is required is a twosheeted Riemann space and the fundamental dipoles can then be explicitly integrated.

It is much simpler to obtain an expression for the Stokes' stream function instead of the velocity potential. Let $T^{(n)}\left(\psi_{0}\right), S, \chi$ be the Stokes' stream functions conjugate to $Q^{(n)}\left(\psi_{0}\right), V, \Phi$ respectively. Corresponding to (4.2), (8.3) and $\Phi=V+z$ we have

$$
\begin{aligned}
T^{(n)}\left(\psi_{0}\right) & =\frac{1}{2 \pi i} \int_{W} \frac{t(\xi)}{2 n} \cot \frac{\xi-\psi_{0}}{2 n} d \xi \\
S & =\frac{1}{2 \pi i} \int_{W} \frac{\pi t(\xi)}{2 \alpha}\left[\cot \frac{\pi(\xi+2 \beta)}{2 \alpha}-\cot \frac{\pi \xi}{2 \alpha}\right] d \xi \\
\chi & =S+r^{2} / 2
\end{aligned}
$$

where $t(\xi)$ is obtained from $t\left(\psi_{0}\right)$ in (3.2) by replacing $\psi_{0}$ by $\xi$, and $r^{2} / 2$ is the stream function conjugate to $z$. In any numerical work these have a much simpler form than the corresponding formulas for the potential.

In the integrand the substitution $\xi=\psi+\zeta$ can be made, and the lower half $W^{\prime}$ of the path of integration can be reduced to the upper half $W_{u}^{\prime}$ by the substitution $\zeta \rightarrow-\zeta$. There results 
$T^{(n)}\left(\psi_{0}\right)=\frac{a^{2} \sinh ^{2} \sigma}{4 \pi n i(s-\tau)^{1 / 2}} \int_{W^{\prime} u} \frac{1}{(s-\cos \zeta)^{3 / 2}} \cdot \frac{\sin (\zeta / n) d \zeta}{\cos \left[\left(\psi-\psi_{0}\right) / n\right]-\cos (\zeta / n)}$.

Consider now the case $n=2$. Make the substitution $\cos (\zeta / 2)=\cosh (\sigma / 2) \cosh \eta$ and evaluate the integral. The result is

$$
\begin{aligned}
T^{(2)}\left(\psi_{0}\right)= & t\left(\psi_{0}\right) \frac{2}{\pi} \arctan \left[\left\{\frac{\cosh (\sigma / 2)+\cos \left[\left(\psi-\psi_{0}\right) / 2\right]}{\cosh (\sigma / 2)-\cos \left[\left(\psi-\psi_{0}\right) / 2\right]}\right\}^{1 / 2}\right] \\
& +\frac{2^{1 / 2} a^{2} \sinh ^{2}(\sigma / 2) \cos \left[\left(\psi-\psi_{0}\right) / 2\right]}{\pi(s-\tau)^{1 / 2}\left[s-\cos \left(\psi-\psi_{0}\right)\right]}
\end{aligned}
$$

where $t\left(\psi_{0}\right)$ is the fundamental dipole in (3.2).

The arc tangent factor in (B.5) illustrates the difference in behavior of $T^{(2)}\left(\psi_{0}\right)$ between $\psi \rightarrow \psi_{0}, \sigma \rightarrow 0$ and $\psi \rightarrow \psi_{0}+2 \pi, \sigma \rightarrow 0$. In the former situation, the factor approaches 1 while in the latter it approaches 0 . The second term in (B.5) just cancels the remaining singularity, with the result that $T^{(2)}\left(\psi_{0}\right)-t\left(\psi_{0}\right)$ is regular as $\psi \rightarrow \psi_{0}$, $\sigma \rightarrow 0$ while $T^{(2)}\left(\psi_{0}\right)$ itself is regular as $\psi \rightarrow \psi_{0}+2 \pi, \sigma \rightarrow 0$.

We may now obtain the stream function for the flow about a figure eight lens with $\alpha=2 \pi / m$. For the stream function $S$ conjugate to $V$ we have a formula analogous to (8.2):

$$
S=\sum_{k=1}^{m}\left[T^{(2)}(4 k \pi / m-2 \beta)-T^{(2)}(-4 k \pi / m)\right] .
$$

The case $m=1$ is especially noteworthy since this corresponds to the flow about spherical shell (exterior angle $2 \pi$ ):

$$
S=T^{(2)}(-2 \beta)-T^{(2)}(0), \quad \alpha=2 \pi \text { (spherical shell). }
$$

For the stream function $\chi$, conjugate to $\Phi$, we have

$$
\chi=T^{(2)}(-2 \beta)+T^{(2)}(2 \pi), \quad \alpha=2 \pi \text { (spherical shell) }
$$

since $r^{2} / 2=T^{(1)}(0)=T^{(2)}(0)+T^{(2)}(2 \pi)$. The flow about a spherical shell has also been obtained by Basset [5] using spherical coordinates.

For the sake of completeness, we include here the formula for $Q^{(2)}\left(\psi_{0}\right)$. It is

$$
\begin{aligned}
Q^{(2)}\left(\psi_{0}\right)= & q\left(\psi_{0}\right) f\left(\psi_{0}\right)+\frac{a}{\pi \sqrt{2}} \frac{(s-\tau)^{1 / 2} \sin \left[\left(\psi-\psi_{0}\right) / 2\right]}{s-\cos \left(\psi-\psi_{0}\right)} \\
& -\frac{a}{2} \csc \left(\psi_{0} / 2\right) f(0)+\frac{a}{4} \tan \left(\psi_{0} / 4\right),
\end{aligned}
$$

where $f\left(\psi_{0}\right)$ is the arc tangent factor of $t\left(\psi_{0}\right)$ in (B.5),

$$
f\left(\psi_{0}\right)=\frac{2}{\pi} \arctan [\quad]
$$

The potential function arising from a pole (instead of a dipole) of suitable strength has the form 


$$
\frac{(s-\tau)^{1 / 2}}{\left[s-\cos \left(\psi-\psi_{0}\right)\right]^{1 / 2}} f\left(\psi_{0}\right) .
$$

In particular if the pole is at infinity $\left(\psi_{0}=0\right)$, its form is

$$
f(0) \text {. }
$$

This last expression is a potential function, equal to 1 at infinity in the first sheet $(\psi=0)$, equal to 0 at infinity in the second sheet $(\psi=2 \pi)$ and having no singularities in the finite.

\section{Appendix C}

Completely separated spheres. Although the case of completely separated spheres can be treated by the method of images described in Section 2, and the virtual mass computed from (7.4), nevertheless it is of interest to trace a closer relationship to the case of intersecting spheres. It will be shown that the integral formulas for lens flow carry over to separated spheres by merely allowing the coordinates $\sigma, \psi$ and the geometrical quantities $a, \beta, \alpha$ to be pure imaginary.

For simplicity we shall discuss only the symmetrical case, the extension to the asymmetrical case being clear. Let the two spheres be of radius A, as indicated in Fig. 13 , moving at unit velocity in the direction of their line of centers. The distance between the centers of the spheres is $2(B-A)$. As in the lens case, set

$$
B=A(1+\cos \beta), \quad a=A \sin \beta
$$

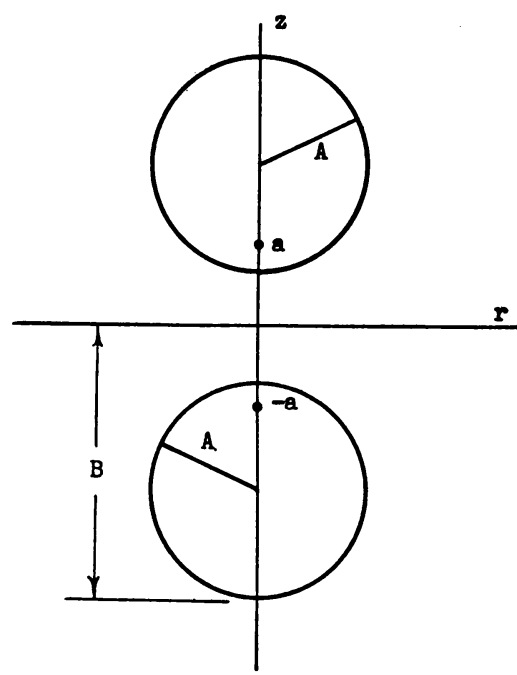

FIG. 13

where now $\beta$ and $a$ are pure imaginary. The points marked $a$ and $-a$ in the diagram are mutually inverse with respect to both spheres. As in the lens case, the dipoles describing the flow are situated at the points $r=0, z=a \cot k \beta$ for $k= \pm 1, \pm 2, \cdots$ with strengths $(-1)^{k+1}\left|a^{3} \csc ^{3}(k \beta) / 2\right|$ respectively.*

The virtual mass $M$ can be obtained from formula (8.4) with the result

$$
m=\frac{M}{\pi \rho A^{3}}=4 \sum_{k=1}^{\infty}(-1)^{k+1} \frac{\sin ^{3} \beta}{\sin ^{3} k \beta}-\frac{8}{3},
$$

where it should be recalled that $\beta$ is imaginary and so the series is rapidly convergent. In particular, if the spheres approach tangency, $\beta=0$ and the result (9.6) is obtained.

As $\beta$ varies from $\pi$ to 0 along the real axis, the spheres first form a thin lens which thickens until the spheres are tangent. Then, as $\beta$ varies from 0 to $i \infty$ along the imaginary axis, the spheres separate and move farther and farther

* We remark that in any problem involving successive inversions relative to two equal spheres, the introduction of $\beta$ by means of (C.1) is a simplifying device. A similar device applies to any two spheres, and will simplify such formulas as in [1], pp. 131-133 and [2], p. 420. This remark also applies to the electrostatic analogue, as in [3], pp. 642-649. 
apart. The virtual mass $m$ and its derivative $d m / d q$ vary continuously, but we see from Fig. 11 that $d^{2} m / d b^{2}$ has a discontinuity at $\beta=0(b=1+\cos \beta=2)$.

We may also obtain the potential function in a form analogous to (6.8) or (6.9). Allow the coordinates $\sigma, \psi$ as well as $a, \beta$ to be pure imaginary. (More generally, complex values of $\sigma, \psi, a, \beta$ may also be selected.) Let $r, z$ be connected with $\sigma, \psi$ by the same relations,

$$
r=\frac{a \sinh \sigma}{\cosh \sigma-\cos \psi}, \quad z=\frac{a \sin \psi}{\cosh \sigma-\cos \psi} .
$$

The coordinates $r, z$ are still real when $\sigma, \psi, a$ are all pure imaginary. In order to have $r \geqq 0$, we suppose that $0 \leqq \operatorname{Im} \sigma \leqq \pi$ while $\operatorname{Im} \psi$ varies from $-\infty$ to $+\infty$. The locus $\psi=-\beta$ is the sphere of radius $A$ and center at $r=0, z=-A \cos \beta$. The coordinates $\operatorname{Im} \sigma, \operatorname{Im} \psi$ and the angle $\theta$ of rotation form the so-called bipolar coordinate system in space.

The function defined by (3.1) remains a potential function even though $\sigma, \psi$ are pure imaginary. The branch points of $q(\xi)$ as a function of the complex variable $\xi$ are still located at $\psi-i \sigma+2 k \pi, \psi+i \sigma+2 k \pi$ but all of these now lie on the line $\operatorname{Im} \xi=\operatorname{Im} \psi$. The $\xi$-plane can be cut along the straight line segments connecting the two adjacent branch points $\psi-i \sigma+2 k \pi$ and $\psi+i \sigma+2(k+1) \pi$ (see Fig. 14). Select the path $\widetilde{W}$ as indicated in Fig. $14 ; \tilde{W}$ can also be considered as surrounding all the individual cuts as well.

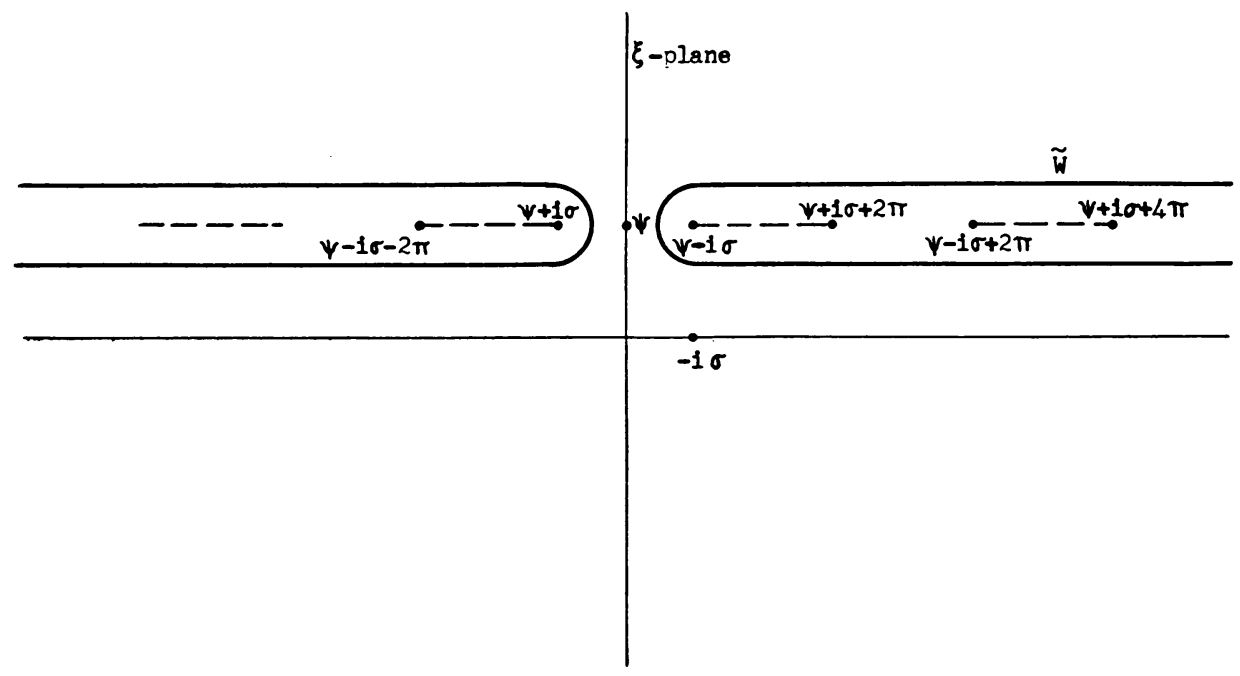

FIG. 14

The potential function $V$ (or $\Phi$ ) can now be constructed as in the lens case, with the same form as in (6.8):

$$
V=-\frac{1}{2 \pi i} \int_{\tilde{W}} q(\xi) \frac{\pi}{2 \beta} \csc \frac{\pi \xi}{2 \beta} d \xi .
$$

The formulas for the virtual mass $M$ is the same as (7.4), (7.7) except that the mass 
of the fluid displaced is merely $\frac{8}{3} \pi \rho A^{3}$. The integral in (7.7) can then be evaluated by residues for $\beta$ pure imaginary, with the result already obtained in (C.2).

\section{REFERENCES}

[1] H. LAMB, Hydrodynamics, 6th ed., MacMillan, 1932.

[2] L. M. Milne-Thomson, Theoretical hydrodynamics, MacMillan, 1939.

[3] P. Frank and R. v. Mises, Die Differential- und Integralgleichungen der Mechanik und Physik, Rosenberg Publ., N. Y. C., 1943, vol. 2, pp. 808-875, by A. Sommerfeld, "Theorie der Beugung."

[4] A. Sommerfeld, Über verzweigte Potentiale im Raum, Proc. London Math. Soc. (1) 28, 395-429 (1897).

[5] Basset, Proc. London Math. Soc. (1) 16, 286 (1885). 